\title{
Determination and Quantification of 5-Hydroxymethylfurfural in Vinegars and Soy Sauces
}

\author{
Li-Li Zhang, Ying Sun, Yu-Yu Zhang, Bao-Guo Sun, and Hai-Tao Chen \\ Beijing Advanced Innovation Center for Food Nutrition and Human Health, \\ Beijing Laboratory for Food Quality and Safety, School of Materials Science and Mechanical Engineering, \\ Beijing Technology \& Business University (BTBU), Beijing 100048, China \\ Correspondence should be addressed to Yu-Yu Zhang; zhangyy2@163.com
}

Received 13 June 2017; Revised 7 July 2017; Accepted 17 July 2017; Published 16 August 2017

Academic Editor: Susana Fiszman

Copyright (C) 2017 Li-Li Zhang et al. This is an open access article distributed under the Creative Commons Attribution License, which permits unrestricted use, distribution, and reproduction in any medium, provided the original work is properly cited.

\begin{abstract}
The organic compound 5-hydroxymethylfurfural (HMF) can be formed from sugars under Maillard reaction and caramelization. In order to study the formation regular of HMF in sugary liquid condiment, vinegar and soy sauce were selected. High-performance liquid chromatography (HPLC) was used to determine the HMF concentrations of various brands of soy sauce and vinegar. The result showed that HMF concentrations were in a range of 0.42 to $115.43 \mathrm{mg} / \mathrm{kg}$ for vinegar samples and 0.43 to $5.85 \mathrm{mg} / \mathrm{kg}$ for soy sauce samples. The concentrates of HMF were expressed in zero-order kinetics model at $100^{\circ} \mathrm{C}$ before the maximum $\mathrm{HMF}$ generation in all of the tested samples. Longer heating treatment time would reduce the HMF content in tested samples. In addition, HMF content had obviously positive correlation with sugar contents in vinegar samples, but no similar rule was found in soy sauces.
\end{abstract}

\section{Introduction}

As typical representatives of liquid condiment, vinegar and soy sauce have gained lots of acceptance and popularity over China for hundred years due to their unique qualities [1].

Vinegar can be fermented from various materials such as rice, glutinous rice, and sorghum. After two steps of fermentation, alcoholic fermentation and acetic fermentation, vinegar was produced [2]. Research has suggested that acetic acid is the main ingredient in vinegar, which gives it an acidic taste [3]. Vinegar is extensively used worldwide as a condiment, acidulant, and food preservative [4]. Every year over 26 million hectoliters of vinegar is produced and more than 3.2 million liters of vinegar is consumed every day in China [5].

Soy sauce is a fermented soybean food. According to the method of manufacture, the naturally brewed method and the acid hydrolysis method were classified [6]. Although the acid hydrolysis method makes the production of soy sauce very cheap, fermented soy sauce was more popular due to its intense umami taste, characteristic aroma, and nutritional value. During the fermentation process, soy protein is enzymatically degraded to amino acids, including glutamic acid and aspartic acid, and wheat polysaccharides are enzymatically degraded to monosaccharides, including glucose [1]. What is more, soy sauce usually contains added caramel, in some cases molasses, to give it a distinctive appearance [7]. The finished product was pasteurized at a rather high temperature $\left(80^{\circ} \mathrm{C}\right)[8]$.

5-Hydroxymethylfurfural (HMF), a food contaminant produced by caramelization and Maillard reaction, is considered as a potential carcinogen for humans (Zou and others 2015). The previous literature has shown that it was concluded that sugary food heated under household cooking conditions could act as an initiator and promoter of colon cancer because of the presence of HMF [9]. The formations of HMF were inevitable in vinegar and soy sauce, which were seen as essential liquid seasonings for home cooking. Theobald et al. [9] also indicated that it was difficult to estimate the HMF content in commercial samples which was prepared in households.

In order to study the formation regular of HMF in liquid condiment, 10 vinegars and 10 soy sauces were selected in the present study, and high-performance liquid chromatography (HPLC) was used to determine the HMF concentrations of all of tested samples. The $\mathrm{pH}$ values, moisture levels, and 
sugar content were investigated, too. This work would provide a promising strategy to reduce the amount of HMF in the thermally treated foodstuffs, in which vinegars and soy sauces were contained.

\section{Materials and Methods}

2.1. Materials. All vinegar (A1-A10) and soy sauce (B1-B10) samples are Chinese-style brewed and were best sellers in Chinese markets in 2017. High purity (>99\%) HMF standard were purchased from Sigma-Aldrich (St. Louis, MO, USA). Methanol (HPLC grade) was provided by Thermo Fisher Scientific (USA). Ultrapure water was purchased from Hangzhou Wahaha Group Co., Ltd. Deionized water was obtained in house.

2.2. Preparation of Heating Treatment Samples. The samples of $30 \mathrm{~g}$ vinegars in $100 \mathrm{~mL}$ flask were heated at $100^{\circ} \mathrm{C}$ from 10 to $60 \mathrm{~min}$, while soy sauces were heated from 20 to $120 \mathrm{~min}$. After the heated time finished, the samples were removed from the bath for determination with HPLC.

2.3. Measurement of $\mathrm{pH}$. The $\mathrm{pH}$ was measured at $20^{\circ} \mathrm{C}$ using a Mettler Toledo S40 SevenMulti ${ }^{\mathrm{TM}} \mathrm{pH}$ meter (Beijing Songxinhongze Technology Co., Ltd., Beijing, China) after appropriately removing the sediment layer. The meter was calibrated with $\mathrm{pH} 4.0$ and 7.0 buffers.

2.4. Measurement of Moisture Levels and Sugar Content. Chinese National Standard methods were employed to evaluate the general components of samples. Moisture level was determined by electrothermal constant-temperature blast drying oven (WG9220A) according to the change in weight after drying for $20 \mathrm{~h}$. The sugar content was determined by thermal ion chromatograph (IC-3000) according to the GB/T22221-2008 Chinese National Standard.

2.5. HMF Analysis. The analysis of HMF was performed using the method proposed by Gökmen and Şenyuva [10] and Gökmen et al. [11] with modifications. Here, a methanol water solution $(5: 95, \mathrm{v}: \mathrm{v})$ was employed rather than an acetonitrile and acetic acid aqueous solution (10:90, v:v), and HMF was well separated at a flow rate of $1.0 \mathrm{~mL} / \mathrm{min}$.

Each of the vinegar and soy sauce samples (20 g) was placed into a $50 \mathrm{~mL}$ centrifuge tube and covered with a cap. The sample was maintained continuously at $4^{\circ} \mathrm{C}$ while being shaken vigorously for $3 \mathrm{~min}$ and then centrifuged for $15 \mathrm{~min}$ at $9600 \mathrm{rpm}$. Then the supernatant solution and methanol $(\mathrm{v}: \mathrm{v}, 1: 1)$ were mixed and shaken for $3 \mathrm{~min}$. The supernatant solution was filtered through a $0.45 \mu \mathrm{m}$ disk filter and stored at $4^{\circ} \mathrm{C}$ until conducting the analysis. All experiments were conducted in triplicate.

The concentration of HMF was obtained by comparing the retention time value and the UV spectrogram with those of the appropriate standards. The peak area values obtained from the various HMF standards were employed to construct a standard curve, as shown in Figure 1(d).
2.6. Statistical Analysis. Analysis of variance (ANOVA), regression analysis (curve fitting), and the calculation of kinetic rate constants were performed using the Microcal Origin 8.0 software (Origin Lab., Northampton, MA, USA). ANOVA test was performed for all experimental runs to determine significance at $95 \%$ confidence interval. All experiments were performed in triplicate.

\section{Results and Discussion}

Figures 1(a), 1(b), and 1(c) depicted the chromatographic separation of HMF in a representative standard solution and in representative commercial vinegar and soy sauce samples, respectively. The chromatograms clearly indicated that HMF was completely resolved from all other components of the vinegar and soy sauce samples. HMF eluted at approximately $(18.899 \pm 0.11) \min (n=10)$ with good retention time reproducibility.

3.1. pH Value, Sugar, and Moisture Content in Vinegars. The $\mathrm{pH}$, sugar, and moisture content in the vinegar samples were listed in Table 1. Vinegar was obtained by a double fermentation process (alcoholic and acetic fermentation) of sugary and starchy substrates [2]. The key ingredient is acetic acid, which gives it an acidic taste, although there may be additions of other kinds of acid like tartaric and citric [12, 13]. Compared with other condiments, vinegar had a lower $\mathrm{pH}$ value. As shown in Table 1, the $\mathrm{pH}$ value of the 10 vinegars was in the range 2.743-3.598. Although the result of the test was similar to what was reported by Lalou et al. (2.83-3.53) [14], there was significant difference between different tested vinegar samples $(p<0.05)$. There were great variations on moisture content in vinegar samples, too. The highest amount of moisture was $98.292 \%$ (sample A10), while the lowest was 89.010\% (sample A8). Previous research had shown that not only $\mathrm{pH}$ value and moisture content but also varieties and contents of sugar have effects on HMF formation [15-17]. And numerous studies have indicated that fructose is the most reactive sugar relative to sucrose and glucose, in the formation of HMF under acidic conditions [18]. Therefore, sugar content was also considered in this study. In Table 1, the sugar data obtained were listed. The main sugars were glucose and fructose in vinegars. As for sucrose, maltose, and lactose, concentrations were very low in vinegars.

3.2. Initial HMF Concentrations of Vinegars. Vinegar has high antioxidant activity, antimicrobial properties, antidiabetic effects, and therapeutic properties [19], which made it widely used as acidic seasoning. As all food matrixes used for the production of vinegar contain sugar, formation of $\mathrm{HMF}$ during either the production process or storage is possible.

The initial HMF concentrations of the 10 vinegars ranged from 0.42 to $115.43 \mathrm{mg} / \mathrm{kg}$ (Figure 3(a)). The concentrations of HMF in vinegar samples have been reported by many scholars, and values ranging from $0 \mathrm{mg} / \mathrm{kg}$ up to an extremely high value of $14,145 \mathrm{mg} / \mathrm{kg}$ have been reported, which was an indicator of different practices exercised by different 


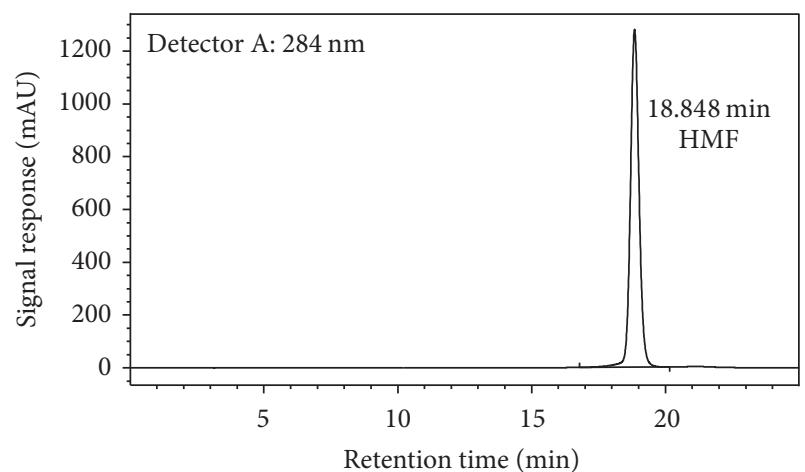

(a)

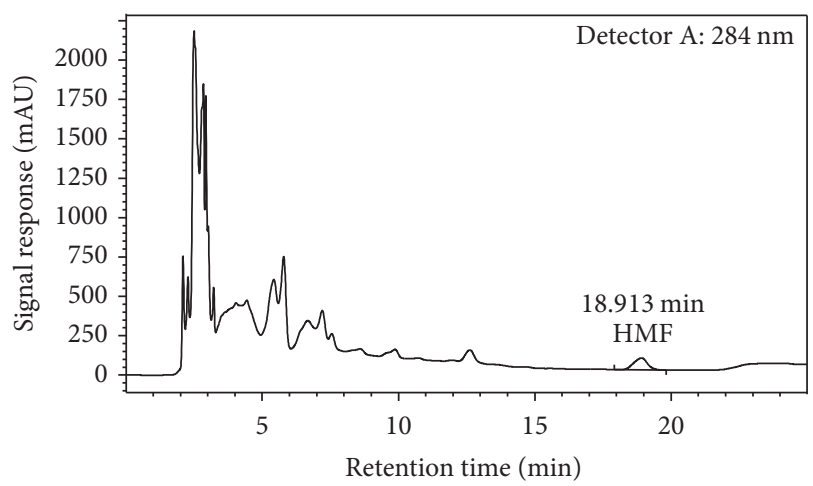

(c)

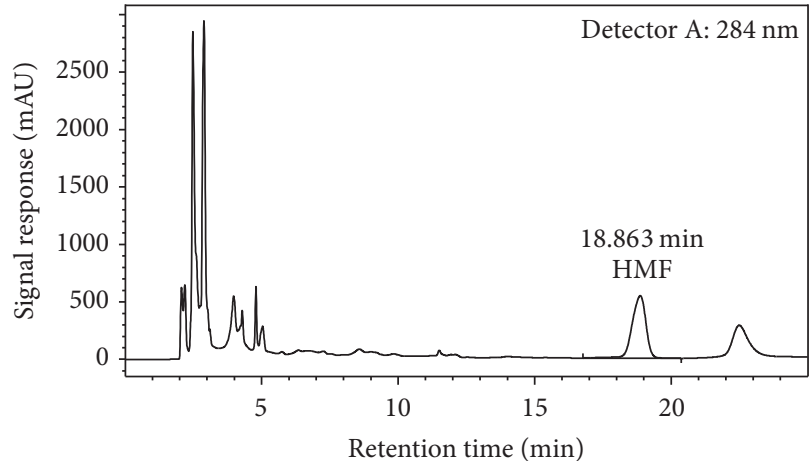

(b)

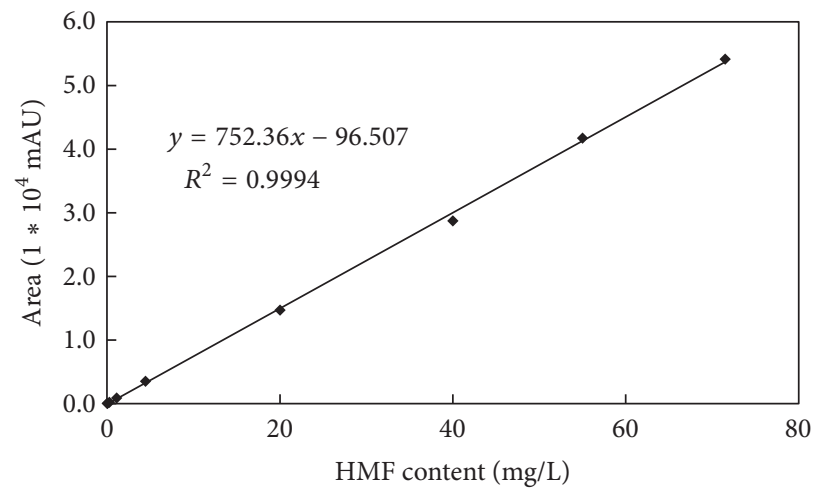

(d)

Figure 1: (a) A representative standard solution. (b) A representative vinegar sample. (c) A representative soy sauce sample; standard 5hydroxymethylfurfural (HMF) (d) determined by high-performance liquid chromatography (HPLC) spectrograms (284 nm).

manufacturers and the lack of consistent process optimization. The range of HMF concentrations for vinegar samples obtained in our study was much lower than that reported by Lalou et al. $(211-14,145 \mathrm{mg} / \mathrm{kg})$ [14]. Caligiani et al. [20] reported a range of HMF concentrations of $0-3.38 \mathrm{mg} / \mathrm{kg}$ for 105 vinegar samples, and they concluded that their method based on proton nuclear magnetic resonance spectroscopy was satisfactory for the quantification of HMF concentration. Bignardi et al. [21] reported a range of HMF concentrations of $0.82-1,153.55 \mathrm{mg} / \mathrm{kg}$ for 3 vinegar samples, which was also greater than that obtained in the present study. In addition, the HMF concentrations reported by Masino et al. [22] for 10 vinegars were found to be significantly greater than the values determined by others. Theobald et al. [9] analyzed the HMF content in various kinds of vinegar. They found that balsamic vinegars exhibited very high HMF concentrations of about $300 \mathrm{mg} / \mathrm{L}$, and, depending on their age, the concentrations of HMF ranged upwards to $5.5 \mathrm{~g} / \mathrm{kg}$.

3.3. HMF Concentrations in Heating Treatment Vinegars. The HMF concentrations in heating treatment vinegars were shown in Figure 3(a), while kinetic analyses of HMF were shown in Table 2. As shown in Figure 3(a), it clearly indicated that HMF concentrations in most of vinegars gradually increased with extension of the heating treatment time. However, HMF concentrates in samples A1, A2, A6, and A9 were showing a trend of increasing first and then decreasing. The highest content of HMF in samples A1 and A6 were $58.28 \mathrm{mg} / \mathrm{kg}$ and $9.08 \mathrm{mg} / \mathrm{kg}$ (40 $\mathrm{min}$ ), and the HMF content was reduced by $2.93 \%$ and $20.70 \%$ when continuously heated for $20 \mathrm{~min}$, respectively. In addition, the time $(50 \mathrm{~min})$ of the highest content of HMF formed was similar in samples A2 and A9. The HMF contents of them were reduced by $2.10 \%$ and $5.96 \%$ when continuously heated for $10 \mathrm{~min}$, respectively. Conclusion was drawn that longer heating treatment time would reduce the HMF content, which might be due to the occurrence of the side reactions of HMF. With Table 2 analysis, the data was expressed in zero-order kinetics model at $100^{\circ} \mathrm{C}$ before the maximum $\mathrm{HMF}$ generation in all of vinegar samples. Among the 10 vinegars, the maximum formation rate of HMF was 1.0961 (sample A8), and the minimum was 0.0361 (sample A10). The cause might be related to moisture and sugar content. Higher sugar content, as well as lower moisture content, had effects on promoting HMF formation.

3.4. Effect of Sugar on HMF Contents in Vinegars. HMF content after thermal treatment for the longest time and the initial sugar content were shown in Figure 2(a). In our study, concentrations of HMF had obviously positive correlation with sugar contents in vinegars. For all of tested vinegars, the highest HMF content was $181.04 \mathrm{mg} / \mathrm{kg}$ (sample A8), followed by $79.53 \mathrm{mg} / \mathrm{kg}$ (sample A9). The highest sugar content was 
TABLE 1: $\mathrm{pH}$ value, sugar, and moisture contents in vinegars and soy sauces.

\begin{tabular}{|c|c|c|c|c|c|c|c|}
\hline Sample & Glucose & Fructose & $\begin{array}{c}\text { Sucrose } \\
(\%)\end{array}$ & Maltose & Lactose & $\mathrm{pH}(n=3)$ & Moisture (\%) $(n=3)$ \\
\hline \multicolumn{2}{|c|}{ Vinegar } & & - & - & - & & \\
\hline $\mathrm{A} 1$ & - & - & - & - & - & $3.317 \pm 0.003(f)$ & $95.028 \pm 0.000(\mathrm{~d})$ \\
\hline A2 & 0.317 & - & - & - & - & $3.411 \pm 0.001(\mathrm{~d})$ & $94.172 \pm 0.001(\mathrm{~g})$ \\
\hline A3 & 0.547 & - & - & - & - & $3.385 \pm 0.001(\mathrm{e})$ & $94.217 \pm 0.000(f g)$ \\
\hline A4 & 0.198 & - & - & - & - & $3.283 \pm 0.002(\mathrm{~g})$ & $95.471 \pm 0.000(\mathrm{c})$ \\
\hline A5 & - & - & - & - & - & $3.248 \pm 0.007(\mathrm{~h})$ & $97.076 \pm 0.000(b)$ \\
\hline A6 & 0.126 & - & - & - & - & $3.598 \pm 0.002(a)$ & $94.753 \pm 0.001(\mathrm{e})$ \\
\hline A7 & 0.262 & - & - & - & - & $3.503 \pm 0.005(b)$ & $90.745 \pm 0.001(\mathrm{~h})$ \\
\hline A8 & 0.990 & 0.293 & - & - & - & $3.478 \pm 0.001(\mathrm{c})$ & $89.010 \pm 0.001(\mathrm{i})$ \\
\hline A9 & 0.677 & - & - & - & - & $3.052 \pm 0.001(\mathrm{i})$ & $94.309 \pm 0.000(f)$ \\
\hline $\mathrm{A} 10$ & 0.218 & 0.240 & - & - & - & $2.743 \pm 0.004(\mathrm{j})$ & $98.292 \pm 0.000(\mathrm{a})$ \\
\hline \multicolumn{2}{|c|}{ Soy sauce } & & & & & & \\
\hline B1 & 0.933 & - & - & - & - & $4.620 \pm 0.006(\mathrm{~g})$ & $70.346 \pm 0.004(\mathrm{~d})$ \\
\hline B2 & 0.444 & - & - & - & - & $4.334 \pm 0.005(\mathrm{j})$ & $74.543 \pm 0.001(\mathrm{a})$ \\
\hline B3 & 1.150 & 0.137 & 4.310 & - & - & $4.895 \pm 0.003(\mathrm{~d})$ & $63.518 \pm 0.001(\mathrm{~h})$ \\
\hline B4 & 0.372 & - & - & - & - & $5.090 \pm 0.003(b)$ & $68.047 \pm 0.001(\mathrm{e})$ \\
\hline B5 & 1.180 & 0.628 & 0.273 & - & - & $4.747 \pm 0.005(\mathrm{e})$ & $71.258 \pm 0.001(\mathrm{c})$ \\
\hline B6 & 1.180 & 0.368 & - & 0.122 & - & $5.018 \pm 0.002(\mathrm{i})$ & $67.351 \pm 0.000(b)$ \\
\hline B7 & 1.380 & 0.367 & - & - & - & $4.456 \pm 0.002(\mathrm{~h})$ & $62.267 \pm 0.001(\mathrm{i})$ \\
\hline B8 & 0.474 & - & 1.060 & - & - & $4.669 \pm 0.001(f)$ & $73.611 \pm 0.000(b)$ \\
\hline B9 & 0.662 & - & - & - & - & $5.122 \pm 0.002(\mathrm{a})$ & $66.595 \pm 0.000(\mathrm{~g})$ \\
\hline $\mathrm{B} 10$ & 0.636 & - & 1.770 & - & - & $4.362 \pm 0.001(\mathrm{c})$ & $73.446 \pm 0.000(\mathrm{f})$ \\
\hline
\end{tabular}

Different letters indicate that there was significant difference between different kinds of vinegar or soy sauce in "pH value" or "moisture contents."

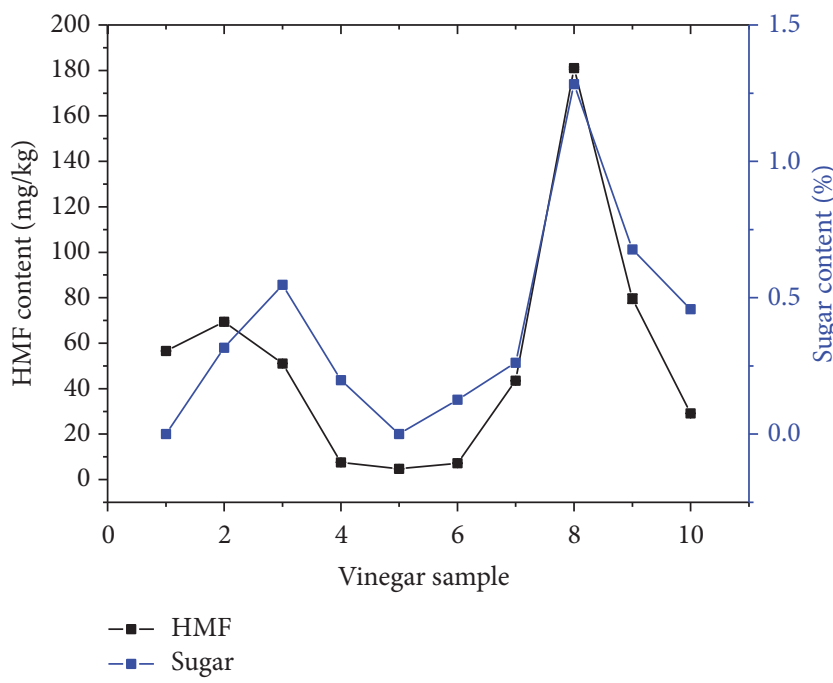

(a)

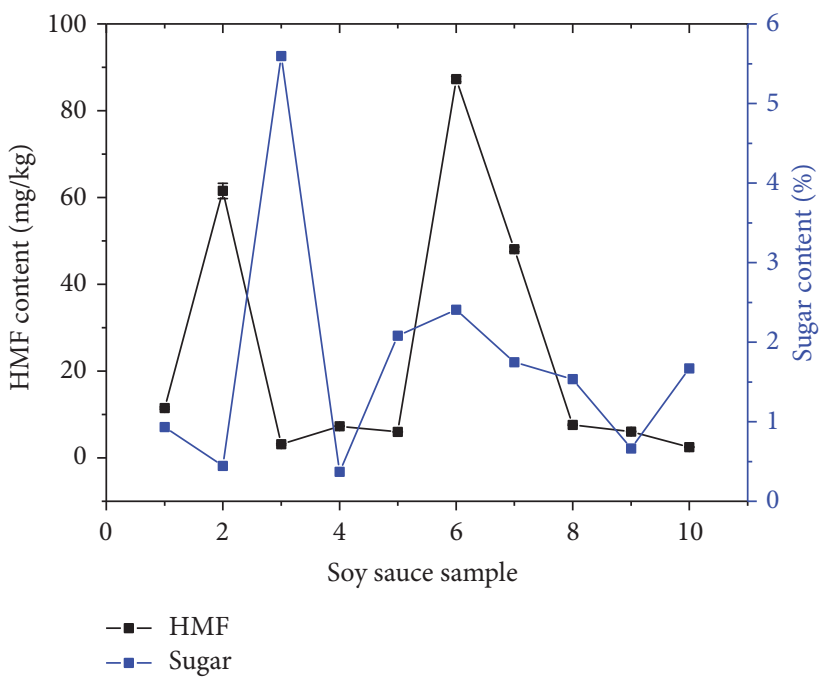

(b)

FIGURE 2: 5-Hydroxymethylfurfural (HMF) and sugar contents in vinegar (a) and soy sauce (b) samples.

$1.283 \%$ (sample A8), where $0.990 \%$ of glucose and $0.293 \%$ of fructose were included. And the second highest sugar content was $0.667 \%$ (sample A9), where only glucose was included. The effects of different kinds of sugar on HMF formation were investigated in sponge cake models under the heating treatment [23]. The result showed that sucrose, lactose, and maltose yielded less HMF than did glucose and fructose. In Locas's study, fructose is the most reactive sugar relative to sucrose and glucose, in the formation of HMF under acidic conditions [17]. These findings were good to support our conclusion that HMF was more readily formed at acid system which was rich in sugars, especially fructose. 


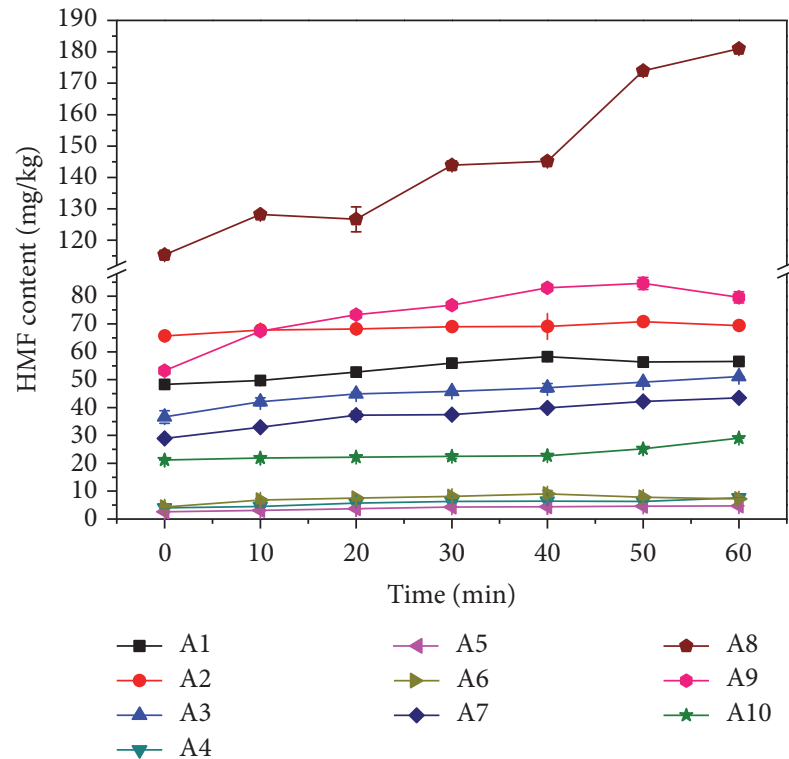

(a)

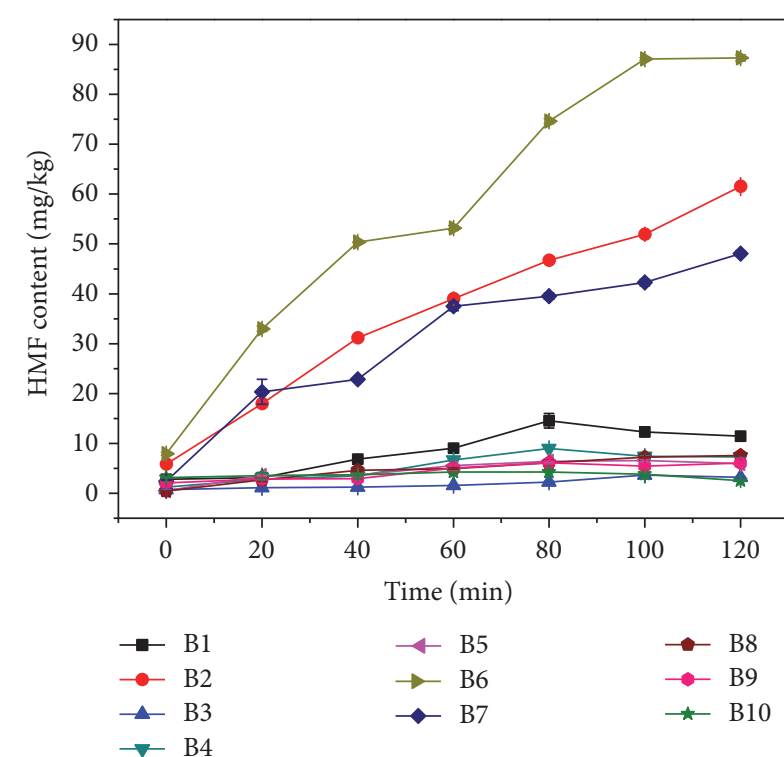

(b)

FIgURE 3: HMF contents of thermal process models (vinegar samples (a), soy sauce samples (b)) at different heating time.

TABLE 2: Summary of kinetic analysis of HMF formation in different heating treatment vinegar and soy sauce samples.

\begin{tabular}{|c|c|c|c|c|c|c|c|}
\hline Sample & Regression equation $^{\mathrm{b}}$ & $K^{\mathrm{c}}$ & $R^{2 \mathrm{~d}}$ & $\mathrm{Af}^{\mathrm{e}}$ & $B f^{f}$ & $S S^{g}$ & RMSE $^{\mathrm{h}}$ \\
\hline \multicolumn{8}{|l|}{ Vinegar } \\
\hline $\mathrm{A} 1$ & $C(t)=0.2622 t+47.735$ & 0.2622 & 0.9862 & 1.0075 & 1.0000 & 0.0004 & 0.4908 \\
\hline $\mathrm{A} 2$ & $C(t)=0.0872 t+66.252$ & 0.0872 & 0.9116 & 1.0095 & 1.0043 & 0.0012 & 0.9763 \\
\hline A3 & $C(t)=0.2123 t+38.887$ & 0.2123 & 0.9243 & 1.0237 & 1.0006 & 0.0063 & 1.3123 \\
\hline A4 & $C(t)=0.0665 t+4.0961$ & 0.0665 & 0.9352 & 1.0419 & 1.0016 & 0.0091 & 0.2768 \\
\hline A5 & $C(t)=0.0364 t+2.8542$ & 0.0364 & 0.9108 & 1.0538 & 1.0037 & 0.0256 & 0.2460 \\
\hline A6 & $C(t)=0.1075 t+5.0323$ & 0.1075 & 0.9019 & 1.0744 & 1.0064 & 0.0390 & 0.5605 \\
\hline A7 & $C(t)=0.2315 t+30.497$ & 0.2315 & 0.9513 & 1.0200 & 1.0008 & 0.0066 & 1.1319 \\
\hline A8 & $C(t)=1.0961 t+112.03$ & 1.0961 & 0.9260 & 1.0387 & 1.0004 & 0.0129 & 6.6946 \\
\hline A9 & $C(t)=0.5909 t+58.271$ & 0.5909 & 0.9123 & 1.0417 & 1.0019 & 0.0143 & 3.4271 \\
\hline $\mathrm{A} 10$ & $C(t)=0.0361 t+21.385$ & 0.0361 & 0.9273 & 1.0062 & 1.0000 & 0.0002 & 0.1598 \\
\hline \multicolumn{8}{|c|}{ Soy sauce } \\
\hline B1 & $C(t)=0.1467 t+1.4035$ & 0.1467 & 0.9267 & 1.2948 & 0.9434 & 0.6056 & 1.3051 \\
\hline B2 & $C(t)=0.4474 t+9.4629$ & 0.4474 & 0.9803 & 1.1226 & 1.0500 & 0.2571 & 2.7391 \\
\hline B3 & $C(t)=0.017 t+0.7038$ & 0.0170 & 0.9257 & 1.0924 & 0.9967 & 0.0403 & 0.1523 \\
\hline B4 & $C(t)=0.0969 t+0.7645$ & 0.0969 & 0.9549 & 1.1903 & 0.9407 & 0.3190 & 0.6660 \\
\hline B5 & $C(t)=0.0693 t+1.1512$ & 0.0693 & 0.9204 & 1.3119 & 1.1279 & 0.8585 & 0.6447 \\
\hline B6 & $C(t)=0.6618 t+16.499$ & 0.6618 & 0.9481 & 1.1991 & 1.0738 & 0.5966 & 6.6933 \\
\hline B7 & $C(t)=0.3526 t+9.2656$ & 0.3526 & 0.9115 & 1.3252 & 1.1543 & 1.9019 & 4.7475 \\
\hline B8 & $C(t)=0.0574 t+1.351$ & 0.0574 & 0.9428 & 1.2553 & 1.1289 & 1.3646 & 0.6107 \\
\hline B9 & $C(t)=0.0514 t+1.7446$ & 0.0514 & 0.9162 & 1.1142 & 0.9959 & 0.0983 & 0.4911 \\
\hline $\mathrm{B} 10$ & $C(t)=0.015 t+3.1703$ & 0.0150 & 0.9453 & 1.0241 & 1.0001 & 0.0033 & 0.1143 \\
\hline
\end{tabular}

$R^{2}, \mathrm{Af}, \mathrm{Bf}, \mathrm{SS}$, and RMSE: indications of reliability and accuracy of models; ${ }^{\mathrm{a}} \mathrm{T}$ : the heating temperature $\left({ }^{\circ} \mathrm{C}\right) ;{ }^{\mathrm{b}} \mathrm{C}(t)$ : the HMF content (mg HMF per kg sample); $t$ : the time of heat treatment $(\mathrm{min}) ;{ }^{\mathrm{c}} k$ : rate constant $\left(\mathrm{min}^{-1}\right) ;{ }^{\mathrm{d}} R^{2}$ : regression coefficient; ${ }^{\mathrm{e}}$ Af: the accuracy factor; ${ }^{\mathrm{f}} \mathrm{Bf}$ : the bias factor; ${ }^{\mathrm{g}} \mathrm{SS}$ : the sum of the squares of the differences of the natural logarithm of observed and predicted values; ${ }^{\mathrm{h}} \mathrm{RMSE}$ : the root mean square error. 
3.5. $p H$ Value, Sugar, and Moisture Content in Soy Sauces. The $\mathrm{pH}$, sugar, and moisture content in soy sauce samples were listed in Table 1. Soy sauce production involved vigorous lactic and alcohol fermentation. During the fermentation process, lactic acid bacteria produce lactic acid and acetic acid, which leads to $\mathrm{pH}$ decrease [1]. As shown in Table 1, the $\mathrm{pH}$ value of the 10 soy sauce samples was in the range 4.334-5.122, which was higher than the highest $\mathrm{pH}$ value (3.598) in 10 vinegar samples. There was significant difference between different soy sauce samples $(p<0.05)$. Lu et al. [24] analyzed the $\mathrm{pH}$ value of 40 samples of Chinese soy sauce; a range of $\mathrm{pH}$ value of 3.86-4.98 was detected. This result was also higher than the $\mathrm{pH}$ value in tested vinegars. The moisture content in soy sauces varied from $62.267 \%$ to $74.543 \%$, which was $26.73 \%$ lower than the moisture content of tested vinegars. In Kim and Lee's study, moisture content in soy sauce showed a decreased trend during fermentation process [25]. That could be one reason that moisture content was so different in different soy sauces. In soy sauce production, soy protein was enzymatically degraded to amino acids, and wheat polysaccharides were enzymatically degraded to monosaccharides [1]. In Table 1 the sugar data obtained in tested soy sauces were listed. The main sugars were glucose, fructose, and sucrose in soy sauces. And maltose was also detected in sample B6. The highest sugar content was 5.597\% (sample B3), in which $1.150 \%$ of glucose, $0.137 \%$ of fructose, and $4.310 \%$ of sucrose were included.

3.6. Initial HMF Concentrations of Soy Sauces. As a condiment, in order for a soy sauce to have palatable taste, about half of its nitrogenous compounds must be free amino acids; in particular, glutamic acid is a very important component [26]. Studies showed that the rates of HMF formation from glucose and sucrose showed enhancement in the presence of the amino acids, especially acidic amino acids [17]. It was inevitable that HMF was formed in soy sauce, which was abundant in sugar and amino acid. The initial HMF concentrations of the 10 soy sauces ranged from 0.43 to $5.85 \mathrm{mg} / \mathrm{kg}$ (Figure 3(a)). No significant differences in the HMF concentrations were observed for sample B5 $(0.49 \pm$ $0.06 \mathrm{mg} / \mathrm{kg})$ and sample B8 $(0.43 \pm 0.01 \mathrm{mg} / \mathrm{kg})$. The highest HMF concentration was obtained for sample B2 (5.85 \pm $0.11 \mathrm{mg} / \mathrm{kg})$, while the lowest was for sample B8 (0.43 \pm $0.01 \mathrm{mg} / \mathrm{kg}$ ). Compared with vinegars, scarce information was available in soy sauce about the content of HMF. Wang et al. [27] reported a range of HMF concentrations of $0-47.56 \mathrm{mg} / \mathrm{kg}$ for 6 kinds of Chinese soy sauce. Goscinny et al. measured the content of HMF in Bouillon sauce, and nonquantifiable traces were observed [28]. In the production processing of soy sauce, it usually contains added caramel, in some cases molasses, to give it a distinctive appearance [7]. Most of the existing literatures focused on the content of HMF in caramel $[29,30]$.

3.7. HMF Concentrations in Heating Treatment Soy Sauces. The HMF concentrations in heating treatment soy sauces were shown in Figure 3(b), while kinetic analyses of HMF were shown in Table 2. As shown in Figure 3(b), it clearly indicated that HMF concentrations in most of soy sauces keep a continued increase trend with extension of the heating treatment time. However, HMF concentrates in samples B1, B3, B4, B5, B9, and B10 were showing a trend of increasing first and then decreasing. The highest content of HMF in samples B1, B4, B9, and B10 were 14.53, 8.99, 6.11, and $4.27 \mathrm{mg} / \mathrm{kg}(80 \mathrm{~min})$, and the HMF content was reduced by $20.10 \%, 18.69 \%, 0.82 \%$, and $41.55 \%$ when continuously heated for $40 \mathrm{~min}$, respectively. In addition, the time $(100 \mathrm{~min})$ of the highest content of HMF formed was similar in samples B3 and B5. The HMF contents of them were reduced by $12.67 \%$ and $9.56 \%$ when continuously heated for $20 \mathrm{~min}$, respectively. The same conclusion with vinegar sample was drawn that longer heating treatment time would reduce the HMF content. With Table 2 analysis, the data was expressed in zero-order kinetics model at $100^{\circ} \mathrm{C}$ before the maximum HMF generation in all of soy sauce samples. Among the 10 soy sauces, the maximum formation rate of HMF was 0.6618 (sample B6), and the minimum was 0.0150 (sample B10). The cause might be related to moisture and sugar content. In addition, previous study showed that the addition of alanine as a catalyst to maltose solution gave rise to an increase in concentrations of HMF [31].

\subsection{Effect of Sugar on HMF Contents in Soy Sauces. HMF} content after thermal treatment for the longest time and the initial sugar content were shown in Figure 2(b). For tested soy sauces, the highest HMF content was $87.29 \mathrm{mg} / \mathrm{kg}$ (sample B6), followed by $61.51 \mathrm{mg} / \mathrm{kg}$ (sample B2). The highest sugar content was $5.597 \%$ (sample B3), where $1.150 \%$ of glucose, $0.137 \%$ of fructose, and $4.310 \%$ of sucrose were included. And the second highest sugar content was $2.081 \%$ (sample B5), where $1.180 \%$ of glucose, $0.628 \%$ of fructose, and $0.273 \%$ of sucrose were included. However, no association was found between the concentration of HMF and the sugar content. The cause might be that the transforming capacity of sugars was different under the conditions of different $\mathrm{pH}$, amino acid, moisture content, and so on.

\section{Conclusions}

The HMF concentrations of 10 vinegars and 10 soy sauces were evaluated by HPLC. Significant differences $(p<0.05)$ were observed in the HMF concentrations of both vinegar and soy sauce samples, which exhibited a wide variability in a range of 0.42 to $115.43 \mathrm{mg} / \mathrm{kg}$ for vinegar samples and 0.43 to $5.85 \mathrm{mg} / \mathrm{kg}$ for soy sauce samples. Except for samples A4, A5, and A6, the HMF concentrations in the vinegar samples were all greater than those in soy sauce samples. The results showed that the HMF concentrations in soy sauce samples were below the maximum amount allowed in Chinese national standards $(40 \mathrm{mg} / \mathrm{kg})$. However, in the tested vinegar samples, only 4 samples were below this level. In our study, concentrations of HMF had obviously positive correlation with sugar contents in vinegar samples. Longer heating treatment time would reduce the HMF content in all of tested samples. The concentrates of HMF were expressed in zero-order kinetics model at $100^{\circ} \mathrm{C}$ before the maximum HMF generation in both vinegar and soy sauce samples. 


\section{Additional Points}

Practical Application. The kinetic approach was used to estimate the HMF content in heating treatment commercial vinegar and soy sauce samples, which could present a complement mechanism to sugary liquid condiment heated under household cooking conditions. The conclusion will eventually be used to provide a promising strategy to reducing the amount of HMF in the thermally treated foodstuffs, in which vinegars and soy sauces were contained.

\section{Conflicts of Interest}

The authors declare that there are no conflicts of interest.

\section{Acknowledgments}

This work was supported by the National Natural Science Foundation of China (no. 31401604) and the National Key R\&D Program (no. 2016YFD0400705).

\section{References}

[1] H. Katayama, Y. Tatemichi, and A. Nakajima, "Simultaneous quantification of twenty Amadori products in soy sauce using liquid chromatography-tandem mass spectrometry," Food Chemistry, vol. 228, pp. 279-286, 2017.

[2] S. Pornpukdeewattana, S. Kerdpiboon, A. Jindaprasert, P. Pandee, M. Teerarak, and W. Krusong, "Upland rice vinegar vapor inhibits spore germination, hyphal growth and aflatoxin formation in Aspergillus flavus on maize grains," Food Control, vol. 71, pp. 88-93, 2017.

[3] Q. Chen, J. Ding, J. Cai, and J. Zhao, "Rapid measurement of total acid content (TAC) in vinegar using near infrared spectroscopy based on efficient variables selection algorithm and nonlinear regression tools," Food Chemistry, vol. 135, no. 2, pp. 590-595, 2012.

[4] M.-J. De la Haba, M. Arias, P. Ramírez, M.-I. López, and M.-T. Sánchez, "Characterizing and authenticating montilla-moriles PDO vinegars using near infrared reflectance spectroscopy (nirs) technology," Sensors (Switzerland), vol. 14, no. 2, pp. 35283542, 2014.

[5] Q. Zhang, S. Zhang, C. Xie et al., "Characterization of Chinese vinegars by electronic nose," Sensors and Actuators, B: Chemical, vol. 119, no. 2, pp. 538-546, 2006.

[6] A. Sano, T. Satoh, T. Oguma, A. Nakatoh, J.-I. Satoh, and T. Ohgawara, "Determination of levulinic acid in soy sauce by liquid chromatography with mass spectrometric detection," Food Chemistry, vol. 105, no. 3, pp. 1242-1247, 2007.

[7] G. M. Kamal, X. Wang, Bin Yuan et al., "Compositional differences among Chinese soy sauce types studied by 13C NMR spectroscopy coupled with multivariate statistical analysis," Talanta, vol. 158, pp. 89-99, 2016.

[8] A. Miyagi, T. Suzuki, H. Nabetani, and M. Nakajima, "Color control of Japanese soy sauce (shoyu) using membrane technology," Food and Bioproducts Processing, vol. 91, no. 4, pp. 507-514, 2013.

[9] A. Theobald, A. Müller, and E. Anklam, "Determination of 5Hydroxymethylfurfural in vinegar samples by HPLC," Journal of Agricultural and Food Chemistry, vol. 46, no. 5, pp. 1850-1854, 1998.
[10] V. Gökmen and H. Z. Şenyuva, "Improved method for the determination of hydroxymethylfurfural in baby foods using liquid chromatography-mass spectrometry," Journal of Agricultural and Food Chemistry, vol. 54, no. 8, pp. 2845-2849, 2006.

[11] V. Gökmen, Ö. Ç. Açar, A. Serpen, and F. J. Morales, "Effect of leavening agents and sugars on the formation of hydroxymethylfurfural in cookies during baking," European Food Research and Technology, vol. 226, no. 5, pp. 1031-1037, 2008.

[12] Z. Wang, T. Li, F. Liu et al., "Effects of ultrasonic treatment on the maturation of Zhenjiang vinegar," Ultrasonics Sonochemistry, vol. 39, pp. 272-280, 2017.

[13] Y. Kong, L. Zhang, Y. Sun, Y. Zhang, B. Sun, and H. Chen, "Determination of the free amino acid, organic acid, and nucleotide in commercial vinegars," Journal of Food Science, vol. 82, no. 5, pp. 1116-1123, 2017.

[14] S. Lalou, E. Hatzidimitriou, M. Papadopoulou et al., "Beyond traditional balsamic vinegar: compositional and sensorial characteristics of industrial balsamic vinegars and regulatory requirements," Journal of Food Composition and Analysis, vol. 43, pp. 175-184, 2015.

[15] M. Anese, F. Bot, and M. Suman, "Furan and 5-hydroxymethylfurfural removal from high- and low-moisture foods," LWT - Food Science and Technology, vol. 56, no. 2, pp. 529-532, 2014.

[16] B. Fallico, M. Zappalà, E. Arena, and A. Verzera, "Effects of conditioning on HMF content in unifloral honeys," Food Chemistry, vol. 85, no. 2, pp. 305-313, 2004.

[17] C. P. Locas and V. A. Yaylayan, "Isotope labeling studies on the formation of 5-(hydroxymethyl)-2- furaldehyde (HMF) from sucrose by pyrolysis-GC/MS," Journal of Agricultural and Food Chemistry, vol. 56, no. 15, pp. 6717-6723, 2008.

[18] H. S. Lee and S. Nagy, "Relative reactivities of sugars in the formation of 5-hydroxymethylfurfural in sugar-catalyst model systems," Journal of Food Processing and Preservation, vol. 14, no. 3, pp. 171-178, 1990.

[19] N. H. Budak, E. Aykin, A. C. Seydim, A. K. Greene, and Z. B. Guzel-Seydim, "Functional properties of vinegar," Journal of Food Science, vol. 79, no. 5, pp. R757-R764, 2014.

[20] A. Caligiani, D. Acquotti, G. Palla, and V. Bocchi, "Identification and quantification of the main organic components of vinegars by high resolution $1 \mathrm{H}$ NMR spectroscopy," Analytica Chimica Acta, vol. 585, no. 1, pp. 110-119, 2007.

[21] C. Bignardi, A. Cavazza, and C. Corradini, "Selected product ion monitoring for quantification of 5-hydroxymethylfurfural in food products by capillary zone electrophoresis-tandem ion trap mass spectrometry," Food Control, vol. 46, pp. 41-48, 2014.

[22] F. Masino, F. Chinnici, A. Bendini, G. Montevecchi, and A. Antonelli, "A study on relationships among chemical, physical, and qualitative assessment in traditional balsamic vinegar," Food Chemistry, vol. 106, no. 1, pp. 90-95, 2008.

[23] Y.-Y. Zhang, Y. Song, X.-S. Hu, X.-J. Liao, Y.-Y. Ni, and Q.-H. Li, "Effects of sugars in batter formula and baking conditions on 5hydroxymethylfurfural and furfural formation in sponge cake models," Food Research International, vol. 49, no. 1, pp. 439-445, 2012.

[24] Y. Lu, X. Chen, J. Mei et al., "Biogenic amines in Chinese soy sauce," Food Control, vol. 20, no. 6, pp. 593-597, 2009.

[25] J.-S. Kim and Y.-S. Lee, "A study of chemical characteristics of soy sauce and mixed soy sauce: chemical characteristics of soy sauce," European Food Research and Technology, vol. 227, no. 3, pp. 933-944, 2008. 
[26] T. Yokotsuka, "Soy sauce biochemistry," Advances in Food Research, vol. 30, pp. 195-329, 1986.

[27] M. F. Wang, S. H. Zhang, X. D. Guo, Z. F. Du, Y. L. Wu, and Y. H. Wang, "Determination of 5-Hydroxymethylfurfural in Soy by HPLC," Modern Food Science and Technology, vol. 24, pp. 188190, 2008 (Chinese).

[28] S. Goscinny, V. Hanot, H. Trabelsi, and J. Van Loco, "Determination of caramel colorants' by-products in liquid foods by ultra-high-performance liquid chromatography-tandem mass spectrometry (UPLC-MS/MS)," Food Additives and Contaminants - Part A Chemistry, Analysis, Control, Exposure and Risk Assessment, vol. 31, no. 10, pp. 1652-1660, 2014.

[29] I. I. Hewala, M. Zoweil, and S. M. Onsi, "Detection and determination of interfering 5-hydroxymethylfurfural in the analysis of caramel-coloured pharmaceutical syrups," Journal of Clinical Pharmacy and Therapeutics, vol. 18, no. 1, pp. 49-53, 1993.

[30] L. Paravisini, A. Prot, C. Gouttefangeas et al., "Characterisation of the volatile fraction of aromatic caramel using heart-cutting multidimensional gas chromatography," Food Chemistry, vol. 167, pp. 281-289, 2015.

[31] H. H. M. Fadel and A. Farouk, "Caramelization of maltose solution in presence of alanine," Amino Acids, vol. 22, no. 2, pp. 199-213, 2002. 

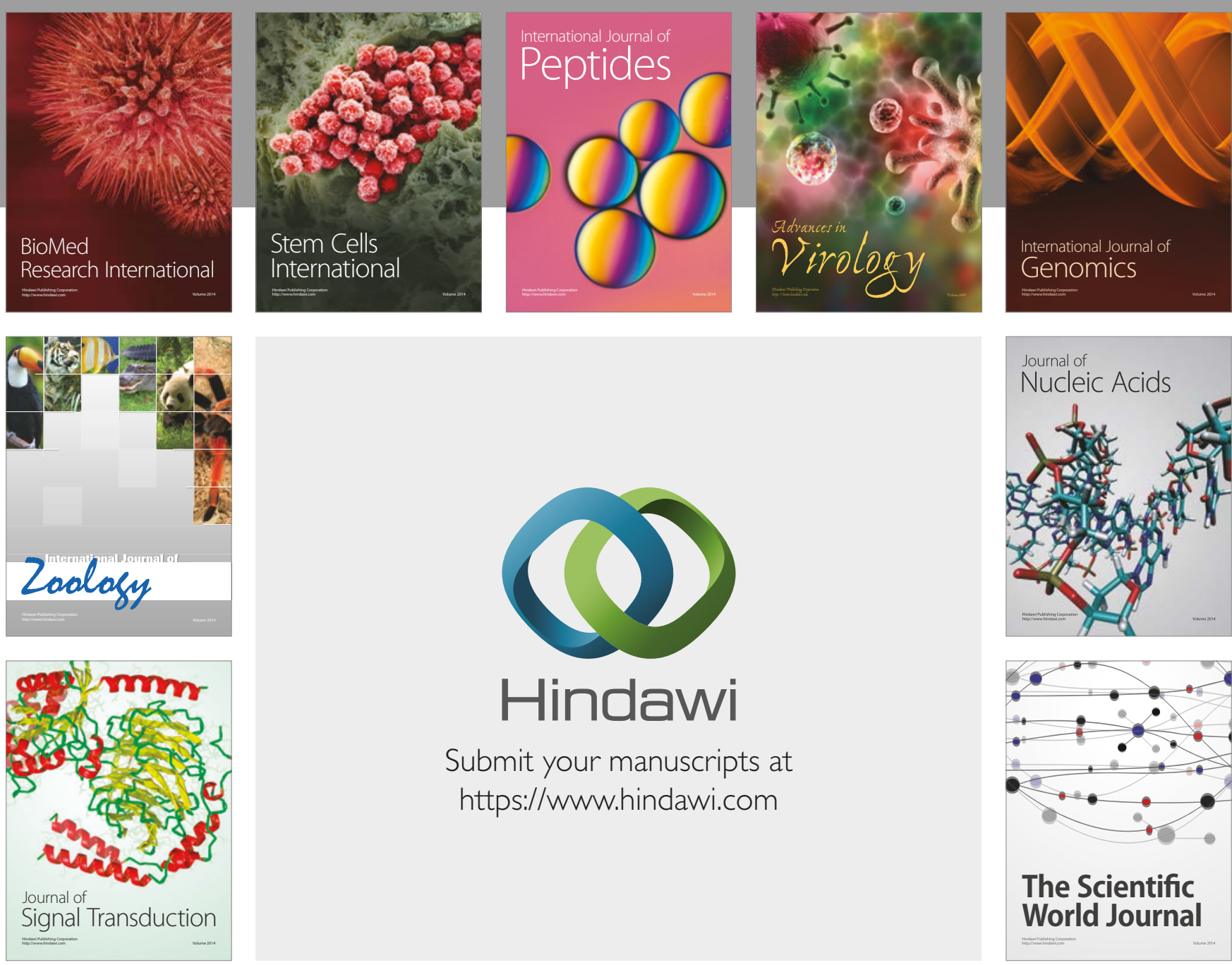

Submit your manuscripts at

https://www.hindawi.com
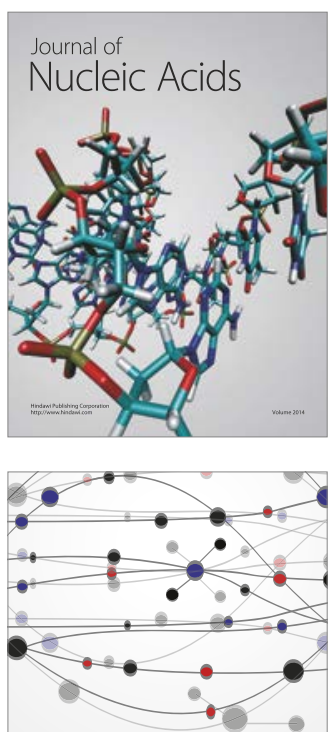

The Scientific World Journal

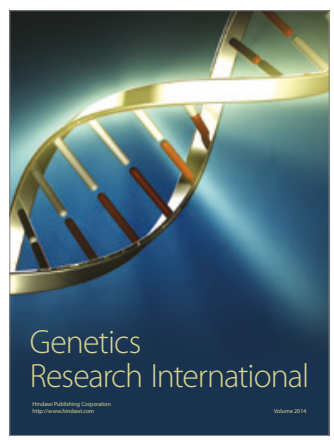

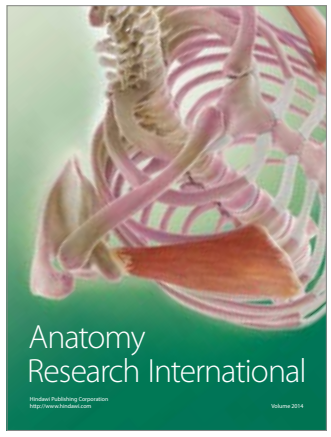

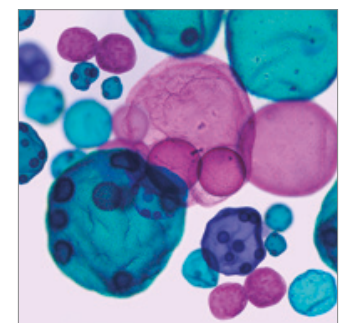

International Journal of Microbiology
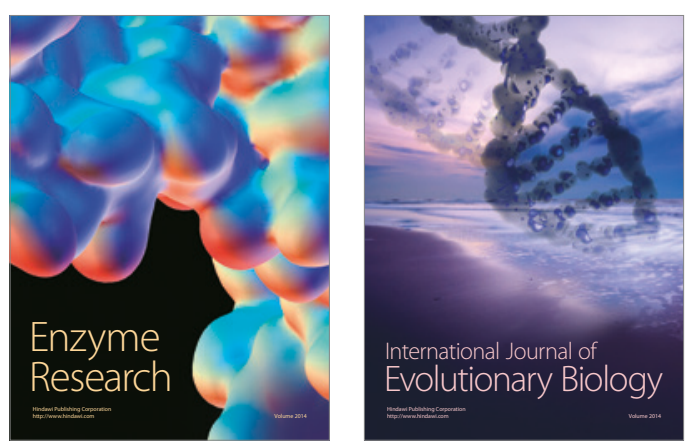
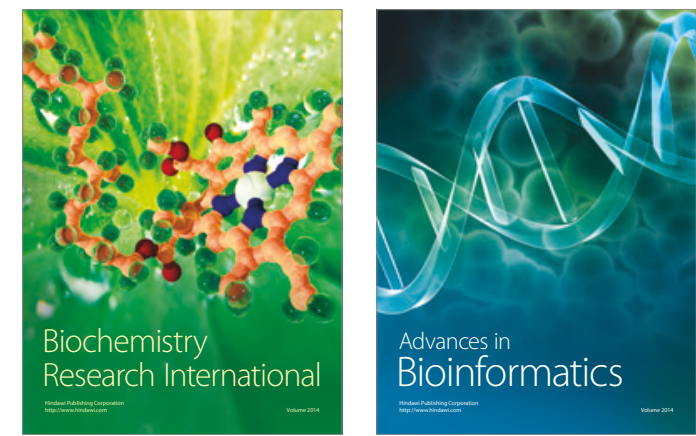

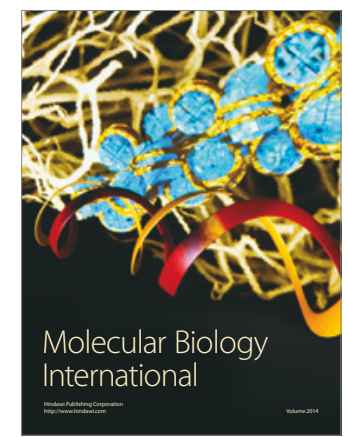

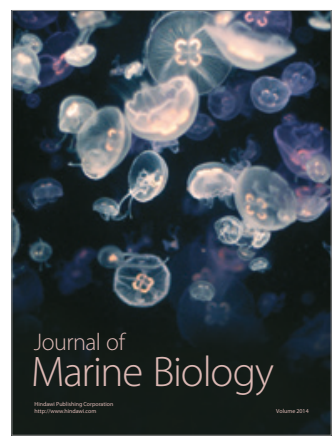

